\title{
Parameterized Sensitivity Analysis of Multiport Systems by means of the Partial Element Equivalent Circuit Method and Interpolation Schemes
}

\author{
L. De Camillis ${ }^{1}$, F. Ferranti ${ }^{1}$, G. Antonini ${ }^{2}$, D. Vande Ginste $^{1}$ and D. De Zutter ${ }^{1}$
}

\begin{abstract}
This paper presents a new methodology to perform parameterized sensitivity analysis based on the electromagnetic method called Partial Element Equivalent Circuit. The proposed methodology is based on the multivariate interpolation of the matrices produced by the electromagnetic solver, which are initially computed at a set of design space points. The proposed interpolation scheme is able to compute the derivatives of the electromagnetic matrices, which are needed to perform the sensitivity analysis. Pertinent numerical results are shown to prove the robustness of the proposed methodology.
\end{abstract}

\section{INTRODUCTION}

The design process of an electromagnetic (EM) structure aims at obtaining the optimal values of the design variables for which the responses of the structure (typically S-parameters in frequency domain or reflection, crosstalk and bit error rate (BER) in time-domain) satisfy the design specifications. This goal is achieved through EM simulations, whereby the desired values of the design variables are usually determined using optimization algorithms (optimizers). These algorithms drive the EM simulators to obtain the responses and their sensitivities in every optimization iteration. Traditional EM-based optimization techniques estimate the responses sensitivities required by the optimizer through a finite-difference approach (FDA). In this approach, the EM simulator is repeatedly invoked for perturbed values of the design variables [1].

Perturbation is a direct, but brute-force, method of sensitivity analysis. It is not only computationally expensive but often inaccurate, thus impractical when the number of circuit parameters for optimization is large. This is because for any circuit parameter $g$, a perturbation $\Delta g$ has to be enforced and a new circuit simulation needs to be run to get the sensitivity with respect to $g$. Furthermore, it may be difficult to select a value for $\Delta g$ to obtain accurate results. If $\Delta g$ is set too large, perturbation itself is inaccurate. If $\Delta g$ is set too small, extremely high accurate simulations are required to exactly highlight the response difference between $g$ and $g+\Delta g$ [2].

This work focuses on a new methodology to perform a parameterized sensitivity analysis based on the Partial
Element Equivalent Circuit (PEEC) [3]. The design parameters involved can be either layout or substrate features of the system under analysis. This method is able to provide sensitivity information over the entire design space and not only around one operative point. This leads to a parameterized state-space model able to perform sensitivity analysis.

The next section briefly reviews the PEEC method. Section 3 introduces the new method of computing sensitivity information over the entire design space, and not only around one operating point. Finally, pertinent numerical results, shown in Section 4, validate the proposed technique.

\section{PEEC METHOD}

The PEEC method [4], [5], [6] is an EM technique, which is able to describe a circuit layout in terms of the capacitive and inductive interactions between the meshcells, and leads directly to a SPICE-compatible model. Nonlinear circuit devices such as drivers and receivers can be connected to PEEC circuits and simulated using a time domain circuit simulator (e.g. SPICE [7]).

In the standard approach, volumes and surfaces of conductors and dielectrics are discretized into hexahedra and patches respectively, that representing elementary regions [8] over which the current and charge densities are expanded into a series of basis functions. The Galerkin's testing technique is used to transform the Electric Field Integral Equation (EFIE) into an equivalent circuit. The circuit equations resulting by enforcing Kirchhoff's current and voltage laws to the PEEC circuit, using the modified nodal analysis (MNA) [9], read:

$$
\left\{\begin{aligned}
\mathbf{C}(\boldsymbol{g}) \dot{\boldsymbol{x}}(t, \boldsymbol{g}) & =-\mathbf{G}(\boldsymbol{g}) \boldsymbol{x}(t, \boldsymbol{g})+\mathbf{B}(\boldsymbol{g}) \boldsymbol{i}_{p}(t, \boldsymbol{g}) \\
\boldsymbol{v}_{p}(t, \boldsymbol{g}) & =\mathbf{L}^{\mathrm{T}}(\boldsymbol{g}) \boldsymbol{x}(t, \boldsymbol{g})
\end{aligned}\right.
$$

where $\boldsymbol{v}_{p}(t, \boldsymbol{g})$ is the port voltage vector, $\boldsymbol{i}_{p}(t, \boldsymbol{g})$ is the port current vector, $\mathbf{C}(\boldsymbol{g}), \mathbf{G}(\boldsymbol{g}), \mathbf{B}(\boldsymbol{g}), \mathbf{L}(\boldsymbol{g})$ are the PEEC matrices, while $\boldsymbol{g}=\left[g_{1}, g_{2}, \ldots, g_{N}\right]$ is the vector of the design parameters of interest.

\footnotetext{
${ }^{1}$ Department of Information Technology, Ghent University, Sint-Pietersnieuwstraat 41, B-9000 Gent, Belgium.

E-mail: \{luca.decamillis, francesco.ferranti, vdginste, daniel.dezutter\}@intec.UGent.be.

${ }^{2}$ Dipartimento Ingegneria Elettrica e dell'Informazione, Via G. Gronchi 18, 67100, L'Aquila, Italy.

E-mail: giulio.antoninieunivaq.it.
} 
Equation (1) represents the system in an impedancebased form that can be recast in the frequency domain as:

$$
\mathbf{V}_{p}(s, \boldsymbol{g})=\mathbf{Z}(\boldsymbol{g}) \mathbf{I}_{p}(s, \boldsymbol{g})
$$

where:

$$
\mathbf{Z}(\boldsymbol{g})=\mathbf{L}^{\mathrm{T}}(\boldsymbol{g})(s \mathbf{C}(\boldsymbol{g})+\mathbf{G}(\boldsymbol{g}))^{-1} \mathbf{B}(\boldsymbol{g})
$$

The sensitivity analysis of (1) can be performed with respect to $\boldsymbol{g}$. The next section describes this in more detail.

\section{Parametric Sensitivity}

When the perturbative approach is used, two EM simulations are required in order to obtain the sensitivity with respect to a design parameter for each point of the design space, and this leads to an inefficient use of the computational resources. In what follows, a new methodology to perform parameterized sensitivity analysis based on the PEEC method is discussed. For easy of notation, we discuss the parameterized sensitivity analysis with respect to one design parameter $g$. The general multivariate case can be easily derived. We derive (1) with respect the parameter of interest $g$, yelding a new state-space system, and combining the result with the original state-space (1) as follows:

$$
\begin{aligned}
& {\left[\begin{array}{ll}
\mathbf{C} & \mathbf{0} \\
\widehat{\mathbf{C}} & \mathbf{C}
\end{array}\right]\left[\begin{array}{l}
\dot{\boldsymbol{x}}(t) \\
\hat{\boldsymbol{x}}(t)
\end{array}\right]=-\left[\begin{array}{cc}
\mathbf{G} & \mathbf{0} \\
\widehat{\mathbf{G}} & \mathbf{G}
\end{array}\right]\left[\begin{array}{l}
\boldsymbol{x}(t) \\
\widehat{\boldsymbol{x}}(t)
\end{array}\right]} \\
& +\left[\begin{array}{cc}
\mathbf{B} & \mathbf{0} \\
\widehat{\mathbf{B}} & \mathbf{B}
\end{array}\right]\left[\begin{array}{c}
\boldsymbol{i}_{p}(t) \\
\widehat{\boldsymbol{i}}_{p}(t)
\end{array}\right] \\
& {\left[\begin{array}{l}
\boldsymbol{v}_{p}(t) \\
\widehat{\boldsymbol{v}}_{p}(t)
\end{array}\right]=\left[\begin{array}{ll}
\mathbf{L} & \widehat{\mathbf{L}} \\
\mathbf{0} & \mathbf{L}
\end{array}\right]^{\mathrm{T}}\left[\begin{array}{l}
\boldsymbol{x}(t) \\
\widehat{\boldsymbol{x}}(t)
\end{array}\right]}
\end{aligned}
$$

where the hat notation $\widehat{\boldsymbol{f}}$ denotes the derivative of the function $f$ with respect to $g$. From (4) it is also possible to derive an impedance-based form:

$$
\left[\begin{array}{l}
\mathbf{V}_{p}(s) \\
\widehat{\mathbf{V}}_{p}(s)
\end{array}\right]=\left[\begin{array}{ll}
\mathbf{Z} & \mathbf{0} \\
\widehat{\mathbf{Z}} & \mathbf{Z}
\end{array}\right]\left[\begin{array}{l}
\mathbf{I}_{p}(s) \\
\widehat{\mathbf{I}}_{p}(s)
\end{array}\right]
$$

where:

$$
\begin{array}{r}
\widehat{\mathbf{Z}}=\widehat{\mathbf{L}}^{\mathrm{T}}(s \mathbf{C}+\mathbf{G})^{-1} \mathbf{B}+\mathbf{L}^{\mathrm{T}}(s \mathbf{C}+\mathbf{G})^{-1} \widehat{\mathbf{B}} \\
-\mathbf{L}^{\mathrm{T}}(s \mathbf{C}+\mathbf{G})^{-1}(s \widehat{\mathbf{C}}+\widehat{\mathbf{G}})(s \mathbf{C}+\mathbf{G})^{-1} \mathbf{B}
\end{array}
$$

Equations (4) and (5) denote the time and frequency domain sensitivity, which are to be properly integrated with termination conditions. Those equations involve the derivative of the PEEC matrices $\widehat{\mathbf{C}}, \widehat{\mathbf{G}}, \widehat{\mathbf{B}}$ and $\widehat{\mathbf{L}}$. In the next section, we will demonstrate how to build a multivariate interpolation model able to shape the PEEC matrices and corresponding derivatives as functions of the design parameters.

\subsection{Parameterized PEEC matrices and corre- sponding derivatives}

The basic idea is to compute the PEEC matrices for a set of design space points, and then to build corresponding parameterized models by means of interpolation schemes, starting from multivariate data samples $\left\{\boldsymbol{g}_{k}, \mathbf{C}\left(\boldsymbol{g}_{k}\right), \mathbf{G}\left(\boldsymbol{g}_{k}\right), \mathbf{B}\left(\boldsymbol{g}_{k}\right), \mathbf{L}\left(\boldsymbol{g}_{k}\right)\right\}_{k=1}^{K_{t o t}}[10]$. The multivariate cubic spline interpolation method [11] is well-known for its stable and smooth characteristics. The computed models are continuous in the first and second order derivatives, allowing to compute the corresponding derivatives of the electromagnetic matrices in (4).

Two data grids are used in the modeling process: an estimation grid and a validation grid. The first one is utilized to build the parameterized models of the PEEC matrices, which lead to a parameterized model of the matrices $\mathbf{Z}$ and $\widehat{\mathbf{Z}}$. The second grid is utilized to assess the interpolation capability of these parameterized models to describe the system under study in points of the design space previously not used for the construction of the models of the PEEC matrices. To clarify the use of these two data grids, we show in Fig. 1 a possible estimation and validation data grid in the case of two design parameters $\boldsymbol{g}=\left(g^{(1)}, g^{(2)}\right)$. The initial set of PEEC matrices (red crosses in Fig. 1) is used to build the multivariate models by interpolation. The other points (blue circles in Fig. 1) are employed as validation points, instead of being used in models generation.

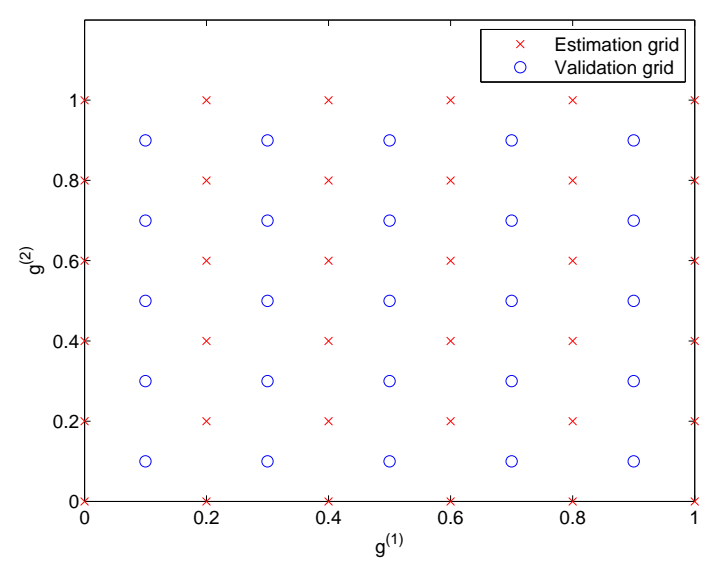

Figure 1. Example of an estimation and validation design space grid.

\section{Numerical EXAMPLE}

The proposed approach is applied to the spiral inductor shown in Fig. 2. The dimensions are $l_{1}=0.8 \mathrm{~mm}$ (length of first edge), $l_{2}=0.96 \mathrm{~mm}$ (length of second edge), $w=0.10 \mathrm{~mm}$ (conductor width), $t=0.05 \mathrm{~mm}$ 


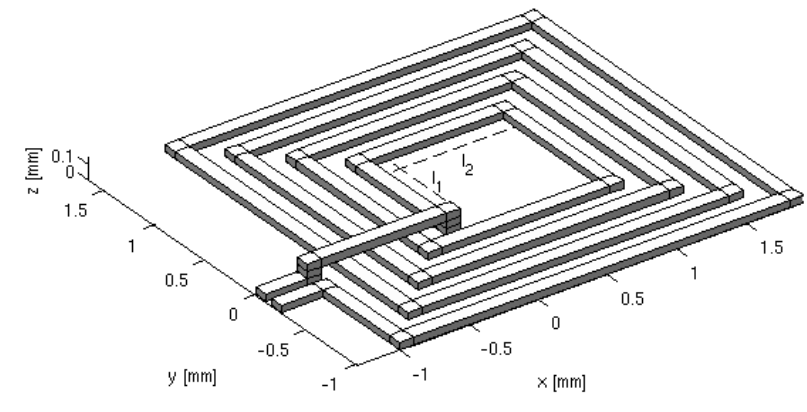

Figure 2. Spiral inductor.

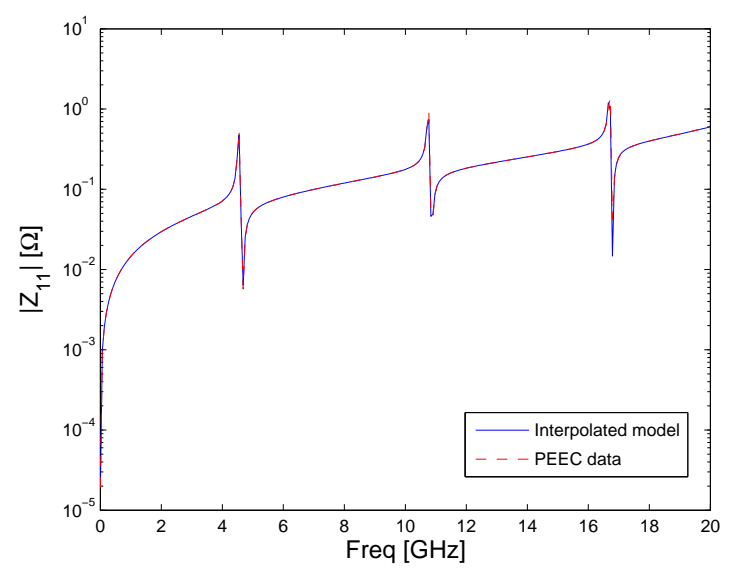

Figure 3. Magnitude spectrum of $\mathrm{Z}_{11}$ for $s=0.14 \mathrm{~mm}$ (validation point).

(thickness of conductor), $n=32$ (number of edges). A parameterized model has been built as a function of the spacing between two successive turns of the spiral $(g=s)$, varying within the interval $[0.10-0.50] \mathrm{mm}$. Six points are used to build the model, while five point are used as validation points. By way of example, the magnitude of $\mathrm{Z}_{11}$ and its derivative are shown in Figs. 3 and 4. Those coefficients have been evaluated for the value of $s=0.14 \mathrm{~mm}$ (validation point). Next, an impulsive voltage source with an internal resistance $R_{T}=50 \Omega$, voltage amplitude equal to $1 \mathrm{~V}$, width of the pulse equal to $5 \mathrm{~ns}$ and rise and fall times equal to $2 \mathrm{~ns}$ is applied to the spiral. This is equivalent to substituting $\left[i_{p}(t) \widehat{i}_{p}(t)\right]^{\mathrm{T}}$ in (4) by:

$$
\left[\begin{array}{c}
i_{p}(t) \\
\hat{i}_{p}(t)
\end{array}\right]=\left[\begin{array}{cc}
1 / R_{T} & 0 \\
0 & 1 / R_{T}
\end{array}\right]\left(\left[\begin{array}{c}
v_{s}(t) \\
0
\end{array}\right]-\left[\begin{array}{c}
v_{p}(t) \\
\widehat{v}_{p}(t)
\end{array}\right]\right)
$$

The port voltage sensitivity, obtained for the five validation points, is shown in Fig. 5 and compared with

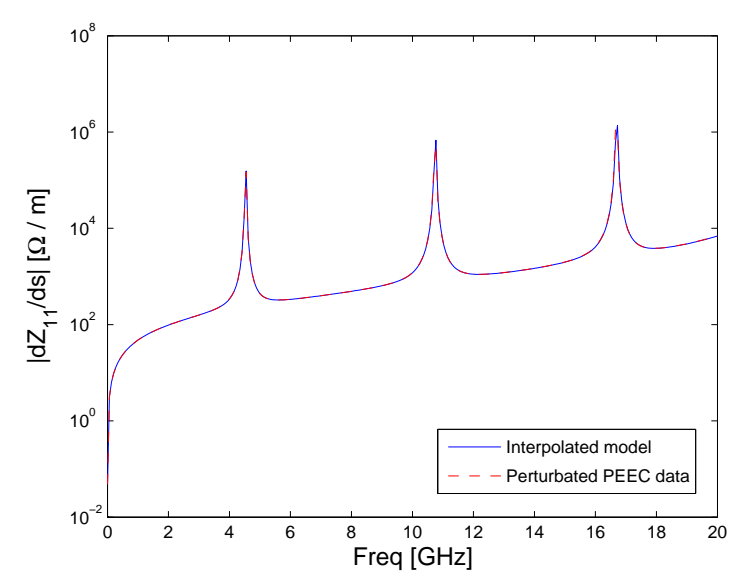

Figure 4. Magnitude spectrum of $\widehat{\mathrm{Z}}_{11}$ for $s=0.14 \mathrm{~mm}$ (validation point).

those results obtained using the perturbative approach:

$$
\widehat{v}_{p}(t)=\frac{v_{p}(t, g+\Delta g)-v_{p}(t, g)}{\Delta g}
$$

The agreement is excellent and demonstrates the accuracy of the proposed methodology.

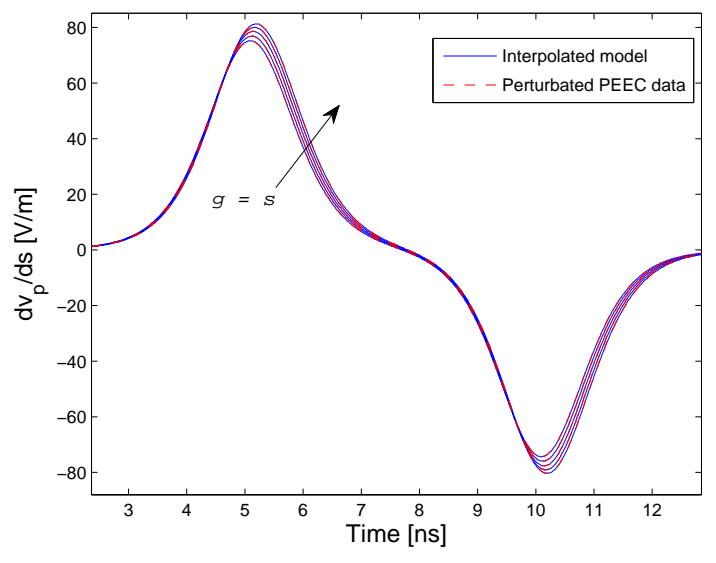

Figure 5. Time domain voltage sensitivity.

\section{Conclusions}

This paper has presented a novel algorithm for parameterized time domain sensitivity analysis, which is based on the combination of the PEEC method and suitable interpolation schemes. The main advantage of the proposed method is that it does not require any perturbative approach and it is able to fully cover the entire design space and not only one operating point. This significantly decreases the computational resources required to carry out a parameterized sensitivity analysis and leads to a more robust and versatile 
approach. A numerical example was provided, which demonstrates the accuracy and modeling capability of the proposed method.

\section{References}

[1] E. A. Soliman, M. H. Bakr, and N. K. Nikolova, "An adjoint variable method for sensitivity calculations of multiport devices," IEEE Transactions on Microwave Theory and Techniques, vol. 52, no. 2, Feb. 2004

[2] J.-F. Mao and E. S. Kuh, "Fast simulation and sensitivity analysis of lossy transmission lines by the method of characteristics," IEEE Transactions on Circuits and Systems, I, vol. 44, no. 5, May 1997.

[3] A. E. Ruehli, P. A. Brennan, "Efficient capacitance calculations for three-dimensional multiconductor systems," IEEE Transactions on Microwave Theory and Techniques, vol. 21, no. 2, pp. 76-82, Feb. 1973

[4] A. E. Ruehli, A. C. Cangellaris, "Progress in the methodologies for the electrical modeling of interconnect and electronic packages," Proceedings of the IEEE, vol. 89, no. 5, pp. 740 771, May 2001.

[5] A. E. Ruehli, "Equivalent circuit models for three dimensional multiconductor systems," IEEE Transactions on Microwave Theory and Techniques, vol. MTT-22, no. 3, pp. 216-221, Mar. 1974.

[6] W. Pinello, A. C. Cangellaris and A. E. Ruehli, "Hybrid electromagnetic modeling of noise interactions in packaged electronics based on the partial-element equivalent circuit formulation," IEEE Transactions on Microwave Theory and Techniques, vol. MTT-45, no. 10, pp. 1889-1896, Oct. 1997.

[7] L. W. Nagel, "SPICE: A computer program to simulate semiconductor circuits," University of California, Berkeley, Electr. Res. Lab. Report ERL M520, May 1975.

[8] A. E. Ruehli, G. Antonini, "On modeling accuracy of EMI problems using PEEC," in Proc. of the IEEE Int. Symp. on Electromagnetic Compatibility, Boston, MA, Aug. 2003.

[9] C. Ho, A. Ruehli, P. Brennan, "The modified nodal approach to network analysis," IEEE Transactions on Circuits and Systems, pp. $504-509$, Jun. 1975.

[10] F. Ferranti, G. Antonini, T. Dhaene, L. Knockaert, and A. Ruehli, "Physics-based passivity-preserving parameterized model order reduction for PEEC circuit analysis," IEEE Transactions on Components, Packaging and Manufacturing Technology, vol. 1, no. 3, pp. 399 - 409, Mar. 2011.

[11] C. De Boor, A practical guide to splines. Springer-Verlag Berlin and Heidelberg GmbH \& Co. K, 1978. 